\title{
Diabetes mellitus and responses of the urinary bladder to acetylcholine: an in vitro study
}

\author{
Abdon M. NSABImana ${ }^{1}$ and John A.O. OJEWOLE ${ }^{1}$ \\ ${ }^{1}$ Department of Pharmacology, School of Pharmacy \& Pharmacology, Faculty of Health \\ Sciences, University of KwaZulu-Natal, Private Bag X54001, Durban 4000, South \\ Africa
}

Received June 8, 2008; Accepted June 27, 2008

\begin{abstract}
This study was prompted by the inconsistent reports and apparent controversies that exist in the biomedical literature on the responses of diabetic bladder strips to cholinergic nerve stimulation or exogenously-administered muscarinic agonists, especially acetylcholine (ACh). In the present study, acetylcholine-induced contractions of urinary bladders isolated from normoglycaemic (normal) and streptozotocin-treated, diabetic Wistar rats were examined under physiological conditions. Mechanical contractile changes of the isolated urinary bladders of STZ-treated, diabetic rats in response to bath-applied acetylcholine were compared with those obtained from isolated urinary bladders of normal, age-matched, control rats. Results obtained show that urinary bladders from diabetic rats were always more spontaneously active after mounting, than those of the age-matched normal, control rats. ACh $\left(10^{-8}-10^{-4} \mathrm{M}\right)$ provoked concentration-related, atropine-sensitive contractions of the isolated urinary bladders of both diabetic and age-matched normal, control rats. However, acetylcholine always induced more powerful and greater contractions of the diabetic bladders compared with bladders from the age-matched normal, control rats. The magnitude and/ or intensity of the diabetic bladder enhanced contractile responses to ACh continued to increase as the diabetic state of the animals progressed.
\end{abstract}

Key words: urinary bladder strips, normal (non-diabetic) and diabetic rats, acetylcholine, contractile response

\section{Introduction}

The mammalian urinary bladder is innervated and functionally regulated by the autonomic nervous system (ANS). Activation of the parasympathetic motor fibres to the bladder causes an intense stimulation of the muscarinic $\mathrm{M}_{3}$-receptors in the bladder body, resulting in a strong and efficient bladder contraction, which in turn causes emptying of the bladder. Urinary bladder

Correspond to: Prof. John A.O. Ojewole, Department of Pharmacology, School of Pharmacy \& Pharmacology, Faculty of Health Sciences, University of KwaZulu-Natal, Private Bag X54001, Durban 4000, South Africa

Phone: +27-31-260-7767/7356 Fax: +27-31-260-7907 e-mail: ojewolej@ukzn.ac.za 
dysfunction is a common complication of chronic diabetes mellitus. Vesical dysfunction is a well-known manifestation of the peripheral autonomic neuropathy that accompanies diabetes mellitus (Kamata et al., 1992). It has been suggested that the initial bladder distension seen in diabetic patients is a consequence of impaired sensory transmission, and sensory neuropathy has been shown to correlate with bladder neuropathy (Hukovic et al., 1965; Kamata et al., 1992). Contractile responsiveness of isolated bladder muscles from streptozotocin (STZ)-treated, diabetic rats to cholinergic nerve stimulation or exogenous administration of muscarinic agonist drugs has been studied by various investigators (Hukovic et al., 1965; Carrier and Aronstam, 1990; Kamata et al., 1992).

Many studies have attempted to clarify the pathogenesis of the urinary tract dysfunction seen in diabetes mellitus (Nakamura et al., 1992). It has been suggested that urinary bladder dysfunction in chronic diabetes might be due to an altered response of the organ to autonomic stimuli (Kamata et al., 1992). Segmental demyelination and axonal degeneration of the peripheral autonomic nerves have also been reported to cause dysfunction of lower urinary bladder tract (van Poppel et al., 1988). Several studies have been undertaken to investigate contractile responses of the bladder to muscarinic drugs in diabetic animals. It has been shown that in diabetic animals, contractile responses of the urinary bladder to muscarinic agonists is inconsistent. Some investigators have reported increased responses (Kolta et al., 1985; Latifpour et al., 1088; Kamata et al., 1992; Tong et al., 2002), while others have reported decreased responses (Longhurst and Belis, 1986; Longhurst et al., 1990), or no change in contractile responses (Lincoln et al., 1984; Luheshi and Zar, 1991) of diabetic urinary bladder smooth muscle strip to muscaric agonists.

The present study was prompted by the existing controversies and inconsistencies in the biomedical literature on the responses of diabetic urinary bladders to muscarinic agonists. The core aim of this study was, therefore, to investigate the responses of urinary bladders isolated from normal (normoglycaemic) and STZ-treated, diabetic Wistar rats to acetylcholine (ACh), a prototype muscarinic agonist.

\section{Materials and methods}

The experimental protocol and procedures used in this study were approved by the Animal Ethics Committee of the University of KwaZulu-Natal, Durban 4000, South Africa; and conform with the "Guide to the Care and Use of Animals in Research and Teaching" (published by the Ethics Committee of the University of Durban-Westville, Durban 4000, South Africa).

\section{Animals and induction of diabetes}

Thirty (30) healthy, young adult, male Wistar rats (Rattus norvegicus) weighing 250-300 g, were used. The animals were kept and maintained under laboratory conditions of temperature, humidity, and light; and were allowed free access to food and water ad libitum one week before the commencement our experiments. The rats were divided into two groups of six (6) normal (non-diabetic) and six (6) STZ-treated, diabetic animals per week following STZ treatment; and were kept in separate cages. Diabetes was induced in the diabetic rats by intraperitoneal 
injections of STZ $(70 \mathrm{mg} / \mathrm{kg})$. The STZ-treated rats were kept in their cages for 7-10 days under laboratory conditions, to allow diabetes to develop in the animals. One to ten week-diabetic rats were used in this study.

\section{Preparation of bladder tissues}

STZ-treated rats with blood glucose concentrations $\geq 15 \mathrm{mmol} / 1$ were considered to be diabetic and used in this study. The normal (non-diabetic) and STZ-treated diabetic rats were sacrificed by decapitation, and the whole (entire) urinary bladder of each rat was removed, opened up longitudinally to form a semi-rectangular piece of tissue, tied with cotton thread at the upper and lower ends, and suspended in 30-ml 'Ugo Basile Two-Chambered Organ Baths' (model 4050) containing Krebs-Henseleit physiological solution (of composition, in mmol/L: $\mathrm{NaCl}, 118.0 ; \mathrm{KCl}, 4.7 ; \mathrm{NaH}_{2} \mathrm{PO}_{4}, 1.28 ; \mathrm{NaHCO}_{3}, 25.0 ; \mathrm{MgCl}_{2}, 1.2 ; \mathrm{CaCl}_{2}, 2.52$; and glucose, 5.55 $\mathrm{pH}$ adjusted to 7.4 ) maintained at $32 \pm 1{ }^{\circ} \mathrm{C}$ and continuously aerated with carbogen (i.e., $5 \%$ carbon-dioxide $+95 \%$ oxygen gas mixture) under an applied resting tension of $1 \mathrm{~g}$. Each bladder muscle strip was allowed to equilibrate for 45-60 min, during which time the bathing physiological solution was changed every $15 \mathrm{~min}$, before it was challenged with graded concentrations of acetylcholine (ACh) (and other drugs used).

\section{Acetylcholine (ACh) Treatment}

Each isolated urinary bladder muscle strip was challenged with graded concentrations of ACh $\left(10^{-8}-10^{-4} \mathrm{M}\right)$ in the absence, and in the presence, of atropine (ATR, $\left.10^{-7}-10^{-5} \mathrm{M}\right)$. The tissues were washed out 3-5 times after the maximal contractile response to each ACh concentration was obtained, and thereafter allowed to equilibrate for 5-10 min before sequential addition of the next higher concentration of acetylcholine. Isolated urinary bladder strips from diabetic and normal, age-matched control animals were always set-up in parallel under the same experimental conditions, in order to make allowance for adequate comparison of the tissues' contractile responses to acetylcholine. The ACh-induced contractile responses of the tissues were recorded isometrically by means of 'Ugo Basile' force displacement transducers and penwriting 'Gemini' recorders (model 7070).

\section{Data analysis}

Data were presented as means $( \pm$ SEM) of the contractile responses of the bladder tissues to the various ACh concentrations. Statistical evaluation of the data was done by using one-way analysis of variance (ANOVA, 95\% confidence interval), and further comparisons done by means of 'Student's $t$-test' for unpaired data. Values of $P \leq 0.05$ were taken to be statistically significant.

\section{Results}

Changes in blood glucose concentrations, body and bladder wet weights

One to ten weeks after treatment with STZ, the fasting blood glucose concentrations of the STZ-treated, diabetic rats were significantly elevated $(P<0.05-0.001)$, compared with those of the normal (non-diabetic), age-matched, control rats (Table 1). Furthermore, the body weights of 
Table 1. Comparison of the fasting blood glucose concentrations (mmol/l) of, and contractile tensions developed ( $\mathrm{g}$ ) by, urinary bladder strips taken from normal (non-diabetic), and STZ-treated diabetic, rats during the ten-week study period. Each value represents the mean $( \pm \mathrm{SEM})$ of six observations

\begin{tabular}{|c|c|c|c|c|}
\hline & \multicolumn{4}{|c|}{ Treatment Groups } \\
\hline & \multicolumn{2}{|c|}{ Diabetic Rats } & \multicolumn{2}{|c|}{ Non-Diabetic (Normal) Rats } \\
\hline & $\begin{array}{c}\text { Blood glucose } \\
\text { level } \\
{[\mathrm{mmol} / \mathrm{l}]}\end{array}$ & $\begin{array}{c}\text { Contractile tension }(\mathrm{g}) \\
\text { developed to } \\
\text { ACh }\left[5.0 \times 10^{-7} \mathrm{M}\right]\end{array}$ & $\begin{array}{c}\text { Blood glucose } \\
\text { level } \\
{[\mathrm{mmol} / \mathrm{l}]}\end{array}$ & $\begin{array}{c}\text { Contractile tension }(\mathrm{g}) \\
\text { developed to } \\
\text { ACh }\left[5.0 \times 10^{-7} \mathrm{M}\right]\end{array}$ \\
\hline Week 1 & $18.5 \pm 1.4^{*} \quad(\mathrm{n}=6)$ & $0.6 \pm 0.02^{*} \quad(\mathrm{n}=6)$ & $4.1 \pm 0.3(n=6)$ & $0.48 \pm 0.07(\mathrm{n}=6)$ \\
\hline Week 2 & $21.3 \pm 1.5^{\star \star} \quad(n=6)$ & $0.9 \pm 0.05^{*} \quad(n=6)$ & $4.0 \pm 0.2(\mathrm{n}=6)$ & $0.43 \pm 0.05(\mathrm{n}=6)$ \\
\hline Week 3 & $24.6 \pm 2.3^{\star \star} \quad(\mathrm{n}=6)$ & $1.3 \pm 0.1^{*} \quad(n=6)$ & $3.96 \pm 0.1 \quad(n=6)$ & $0.45 \pm 0.06(n=6)$ \\
\hline Week 4 & $27.1 \pm 2.4^{\star \star} \quad(n=6)$ & $1.6 \pm 0.2^{\star *} \quad(\mathrm{n}=6)$ & $4.2 \pm 0.3(n=6)$ & $0.46 \pm 0.08(n=6)$ \\
\hline Week 5 & $29.3 \pm 2.7^{* \star *}(\mathrm{n}=6)$ & $1.8 \pm 0.3^{* *} \quad(\mathrm{n}=6)$ & $3.7 \pm 0.1(n=6)$ & $0.44 \pm 0.03(\mathrm{n}=6)$ \\
\hline Week 6 & $31.5 \pm 3.1^{\star \star \star}(\mathrm{n}=6)$ & $2.1 \pm 0.6^{\star \star *}(\mathrm{n}=6)$ & $4.0 \pm 0.2(\mathrm{n}=6)$ & $0.51 \pm 0.05(\mathrm{n}=6)$ \\
\hline Week 7 & $33.7 \pm 3.3^{\star \star \star}(\mathrm{n}=6)$ & $2.5 \pm 0.4^{* * *}(\mathrm{n}=6)$ & $4.1 \pm 0.5(n=6)$ & $0.47 \pm 0.04(\mathrm{n}=6)$ \\
\hline Week 8 & $35.2 \pm 3.8^{\star \star *}(\mathrm{n}=6)$ & $2.8 \pm 0.3^{\star * *}(\mathrm{n}=6)$ & $4.1 \pm 0.4(\mathrm{n}=6)$ & $0.50 \pm 0.03(n=6)$ \\
\hline Week 9 & $36.1 \pm 3.9^{* \star *}(\mathrm{n}=6)$ & $3.0 \pm 0.7^{* * *}(\mathrm{n}=6)$ & $3.8 \pm 0.2(\mathrm{n}=6)$ & $0.49 \pm 0.05(n=6)$ \\
\hline Week 10 & $36.0 \pm 3.7^{* \star *}(\mathrm{n}=6)$ & $3.1 \pm 0.6^{\star * \star} \quad(n=6)$ & $4.0 \pm 0.2(n=6)$ & $0.45 \pm 0.04(\mathrm{n}=6)$ \\
\hline
\end{tabular}

${ }^{\star} P<0.05 ;{ }^{\star \star} P<0.01 ;{ }^{* \star \star} P<0.001$ vs. non-diabetic control rats.

the STZ-treated, diabetic rats decreased significantly $(P<0.05-0.01)$ after six weeks of STZ treatment, compared with the normal (non-diabetic), age-matched, control rats. The bladders of the STZ-treated, diabetic rats were visibly more distended and larger than those of the normal, age-matched control rats at the time of dissection. Moreover, the wet weights of the bladders from diabetic rats were significantly greater $(P<0.05-0.01)$ than those from normal (nondiabetic), age-matched control rats (data not shown).

\section{Acetylcholine-induced contractile responses of the isolated bladders}

Acetylcholine (ACh, $10^{-8}-10^{-4} \mathrm{M}$ ) caused concentration-dependent contractions of bladder strips isolated from both non-diabetic and STZ-treated, diabetic rats. However, acetylcholine always induced stronger and greater contractions of the diabetic bladders compared with bladders taken from the non-diabetic, age-matched control rats. Table 1 compares tensions developed $(\mathrm{g})$ by urinary bladder strips isolated from STZ-treated diabetic, and non-diabetic (normal), rats to a fixed concentration of ACh $\left(5.0 \times 10^{-7} \mathrm{M}\right)$. Figure 1 illustrates typical traces obtained, while Fig. 2 summarizes the results obtained with graded concentrations of ACh $\left(10^{-8}\right.$ $10^{-3} \mathrm{M}$ ) on urinary bladder strips taken from STZ-treated diabetic, and non-diabetic, rats (during week one of our study period). The enhanced contractile responses of the bladder strips to bathapplied ACh were detected soon after induction of diabetes. The magnitude and/or intensity of the enhanced contractile responses of diabetic bladder strips to ACh continued to increase steadily and linearly as the diabetic state of the animals progressed from week one to week ten of the study period (see Table 1). Urinary bladder strips taken from diabetic rats were found to possess greater and more profound intrinsic, spontaneous contractions than their counterparts isolated from normal (non-diabetic) rats (data not shown). Atropine $\left(10^{-7}-10^{-5} \mathrm{M}\right)$, a muscarinic receptor antagonist, competitively reduced or inhibited the contractile responses of the isolated bladder preparations to bath-applied acetylcholine in a concentration-dependent manner. The 


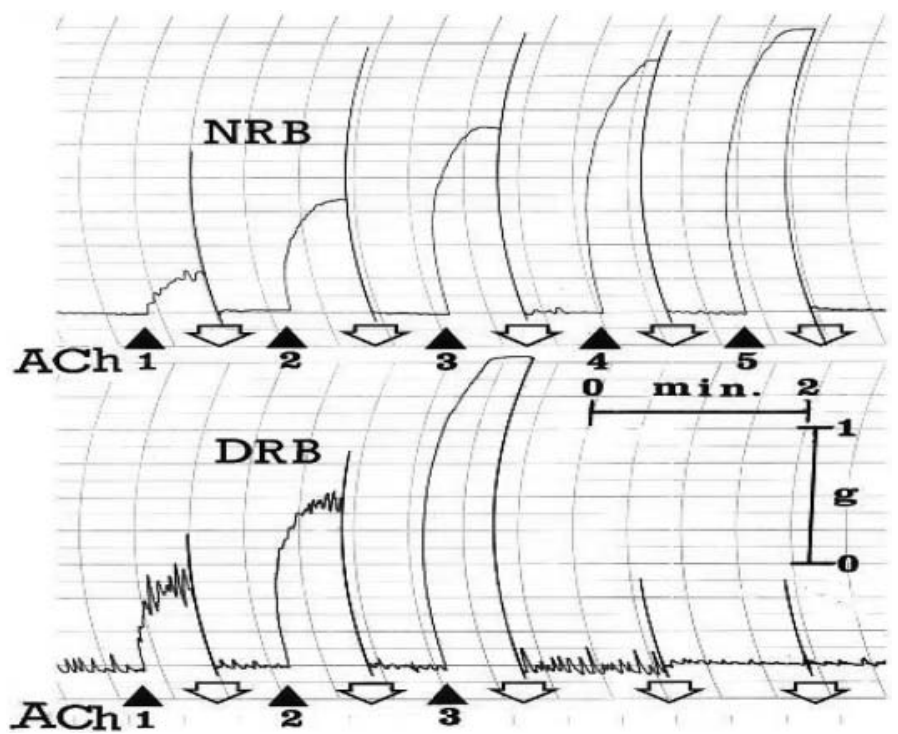

Fig. 1. Effects of graded concentrations of acetylcholine (ACh) on urinary bladder strips taken from a normal (non-diabetic) rat (i.e., NRB, upper trace), and a STZ-treated diabetic rat (i.e., DRB, lower trace), respectively. ACh $\boldsymbol{\Delta} 1,2,3,4$ and 5 denote acetylcholine, $5.0 \times 10^{-8}, 5.0 \times 10^{-7}, 5.0 \times 10^{-6}, 5.0 \times 10^{-5}$ and $5.0 \times 10^{-4} \mathrm{M}$ respectively, sequentially added to the bath-fluid at the solid dots $(\mathbf{A})$, and washed out 3-5 times at the adjacent, open, right-hand-side, downward-pointing arrows.

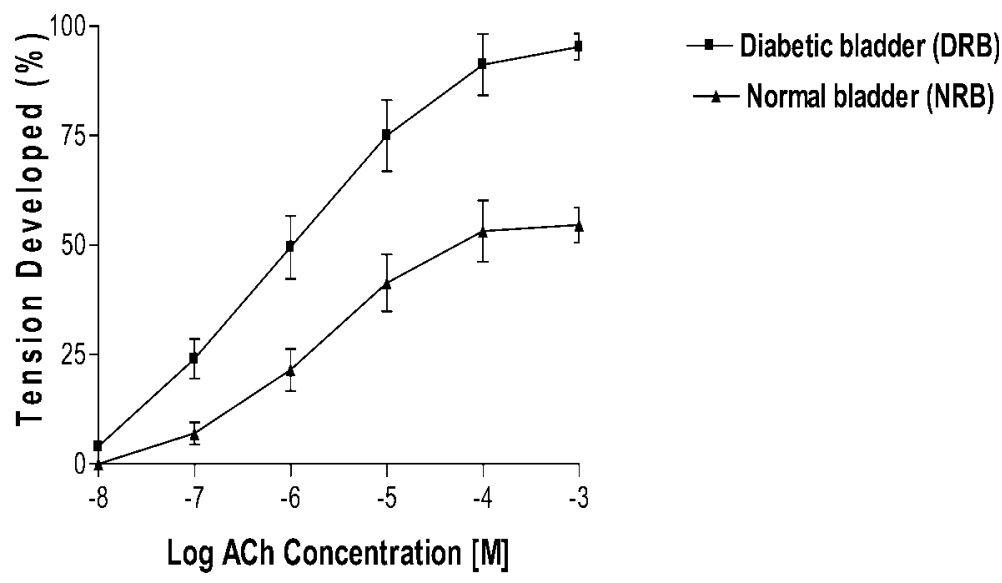

Fig. 2. Comparative tensions developed by urinary bladder strips taken from normal ( $\mathbf{A}$, $\mathrm{NRB})$, and diabetic $(\square, \mathrm{DRB})$, rats to graded concentrations of $\mathrm{ACh}\left(10^{-8}-10^{-3} \mathrm{M}\right)$ (during week one of the study period). Each point represents the mean of six preparations, while the vertical bars denote standard error (SEM) of the mean.

mean $\mathrm{EC}_{50}$ values for $\mathrm{ACh}$ on the isolated bladders of diabetic and age-matched, non-diabetic control rats were found to be $0.01 \pm 0.08 \times 10^{-6} \mathrm{M}(\mathrm{n}=15)$ and $7.21 \pm 0.10 \times 10^{-5} \mathrm{M}(\mathrm{n}=15)$, respectively. 


\section{Discussion}

The results of this animal study show that acetylcholine (ACh) induced stronger and greater contractions of isolated bladders from STZ-treated, diabetic rats compared with bladders isolated from normal (non-diabetic), age-matched, control rats.

It is now firmly established that the sympathetic and parasympathetic nervous systems of the autonomic nervous system (ANS) regulate the functions of the lower urinary bladder in different ways. During the storage phase, the roles of the sympathetic nervous system are relaxation of the bladder body, and contraction of the bladder base through $\alpha$ - and $\beta$ adrenoceptors, respectively; whereas, the role of the parasympathetic nervous system is a contraction of the detrusor muscle through stimulation of the muscarinic $\mathrm{M}_{3}$-receptors in the bladder body to expel urine (Frimodt-Moller, 1976; Nakamura et al., 1992). Lincoln et al. (1984) showed significant increases in the activities of choline acetyltransferase and choline esterase in the bladder after two to eight weeks of diabetes, suggesting that cholinergic nerve activity is increased in the urinary bladder during diabetes mellitus. Lincoln et al. (1984) further observed that norepinephrine content in the bladder showed a tendency to decrease in diabetes.

Previous investigators have shown that in diabetic animals, the contractile response of the urinary bladder to muscarinic agonists is inconsistent. Some investigators have reported increased contractile responses (Kolta et al., 1985; Katifpour et al., 1988; Kamata et al., 1992), while others have reported either decreased responses (Longhurst and Belis, 1986), or no change in contractile responses (Lincoln et al., 1984; Luheshi and Zar, 1991; Nakamura et al., 1992) of the urinary bladder smooth muscle to muscaric agonists. Latifpour et al. (1988) and Tong et al. (2002) have also reported an increased number of muscarinic $\mathrm{M}_{3}$-receptors associated with increased contractile responses to muscarinic agonists in the bladder dome of STZ-induced diabetic rats. On the contrary, Carrier and Aronstam (1990) have reported an increased muscarinic responsiveness and a decreased density of muscarinic receptors in ileal smooth muscles from STZ-treated, diabetic rats. Kamata et al. (1992) have also shown that detrusor strips of urinary bladders from STZ-treated diabetic rats exhibit an enhanced contractile response to acetylcholine, and associated the increased responses to ACh with an increased population of muscarinic receptors in the tissues. However, our results are in agreement with the findings of Kolta et al. (1985), Latifpour et al. (1988) and Kamata et al. (1992) who have reported increased contractile responses of diabetic bladders to muscarinic agonists. The discrepancies in the findings of the various earlier investigators who have examined contractile responses of isolated urinary bladders from diabetic mammals to ACh (or parasympathetic nerve stimulation) are unlikely to be due to differences in species, strains and ages of the experimental animals used because our unpublished, preliminary studies have shown that the enhanced, increased contractile responses of isolated bladders from all available diabetic species and strains of mice, rats, guinea-pigs and rabbits of different ages to bathapplied acetylcholine were not modified by the species, strains and ages of the experimental animals. However, the duration of hyperglycaemia, the segment of the bladder used, the breeders of the animals and the experimental conditions employed, might contribute significantly to the differences in the findings of the earlier investigators. Nilvebrant et al. 
(1986) and Tong and Cheng (2002) noted a "supersensitivity" to muscarinic agonists, and an increased density of muscarinic $\mathrm{M}_{3}$-receptors. Nilvebrant et al. (1986) also noted no change in affinity for ${ }^{3} \mathrm{H}-\mathrm{QNB}$ with hypertrophied bladders following peripheral parasympathetic denervation of the urinary bladders. Latifpour et al. (1988) have reported similar alterations in parasympathetic activities in urinary bladders of diabetic rats. Latifpour et al. (1988) have also shown that STZ-induced diabetic state causes an increased maximum contractile response to muscarinic agonist, an increased density of muscarinic receptors, and no changes in the affinity of the bladder dome smooth muscle for ${ }^{3} \mathrm{H}-\mathrm{QNB}$. However, in their study, Kamata et al. (1992) found that muscarinic receptors in the detrusor strips from STZ-treated diabetic rats had a lower affinity for ${ }^{3} \mathrm{H}-\mathrm{QNB}$ than the detrusor strips from normal, control rats. The latter investigators concluded that although it is unclear why the affinity for ${ }^{3} \mathrm{H}-\mathrm{QNB}$ was lower in diabetic state, it is unlikely that the decreased affinity of muscarinic receptors to acetylcholine is related to the increased contractile response to acetylcholine.

The findings of the present study are in agreement with the works of Kolta et al. (1985) and Tammela et al. (1996) who observed enhanced and significantly greater responses of bladder strips from diabetic rats to acetylcholine and carbachol respectively, soon after induction of diabetes with STZ. They further observed that the magnitude and/or intensity of the acetylcholine- and carbachol-induced contractile responses of the diabetic bladder strips continued to increase as the diabetic state of the animals progressed (as we also observed in the present study) (Tammela et al., 1995; Tong et al., 1996). The increased diabetic bladder mass observed in the present study is also in consonance with the findings of Tammela et al. (1995) who found a significantly increased diabetic bladder mass in their study, and concluded that effects of diabetes (and sucrose consumption) on contractile bladder function are related to diuresis-induced increases in bladder mass.

In conclusion, the findings of the present study have demonstrated that urinary bladders from STZ-treated diabetic rats exhibit an increased contractile responsiveness to bath-applied acetylcholine. Although this preliminary study could not establish the mechanism of the increased contractile responsiveness of diabetic bladders to the muscarinic agonist (ACh) used, our results tend to suggest that the alterations in diabetic urinary bladder synaptosomal, vesiclebound neurotransmitter (ACh) concentrations reported by Tong et al. (1996) and the compensatory increase in the density of muscarinic $\mathrm{M}_{3}$-receptor population reported by Latifpour et al. (1988) would appear to be two of the most attractive, plausible mechanisms of the increased diabetic bladder responsiveness to acetylcholine.

\section{Acknowledgements}

The authors are grateful to Miss K. Moodley for her technical assistance, and to Prof. C. T. Musabayane for his constructive criticisms.

\section{References}

Carrier, G.O. and Aronstam, R.S. (1990). Increased muscarinic responsiveness and decreased 
muscarinic receptor content in ileal smooth muscle in diabetes. J. Pharmacol. Exp. Ther. 254: 445-449.

Frimodt-Moller, C. (1976). Diabetic cystopathy: a clinical study of the frequency of bladder dysfunction in diabetes. Danish Med. Bull. 23: 267-294.

Hukovic, S., Rand, M.J. and Vanor, S. (1965). Observations on isolated innervated preparation of the rat's urinary bladders. Br. J. Pharmacol. 24: 178-183.

Kamata, K., Inoue, K. and Kasuya, Y. (1992). Changes in muscarinic responsiveness, muscarinic receptor density and $\mathrm{Ca}^{2+}$ mobilization of urinary bladder in streptozotocin-induced diabetic rats. Res. Commun. Chem. Pathol. Pharmacol. 75: 143-158.

Kolta, M.G., Wallace, L.J. and Gerald, N.C. (1985). Streptozotocin-induced diabetes affects rat's urinary bladder response to autonomic agents. Diabetes 34: 917-921.

Latifpour, J., Gousse, A., Kondo, S., Morita, T. and Weiss, R.M. (1988). Effects of experimental diabetes on biochemical and functional characteristics of bladder muscarinic receptors. J. Pharmacol. Exp. Therap. 248: 81-88.

Lincoln, J., Crockett, M., Haven, A.J. and Burnstock, G. (1984). Rat bladder in the early stages of streptozotocin-induced diabetes: adrenergic and cholinergic innervations. Diabetologia 26: 8187.

Longhurst, P.A. and Belis, J.A. (1986). Abnormalities of rat bladder in contractility in streptozotocininduced diabetes mellitus. J. Pharmacol. Exp. Therap. 238: 773-777.

Longhurst, P.A., Kang, J., Wein, A.J. and Levin, R.M. (1990). Length-tension relationship of urinary bladder strips from streptozotocin-diabetic rats. Pharmacol. 40: 110-121.

Luheshi, G.N. and Zar, M.A. (1991). The effect of streptozotocin-induced diabetes on cholinergic motor transmission in the rat urinary bladder. Br. J. Pharmacol. 103: 1657-1662.

Nakamura, I., Takahashi, C. and Miyagawa, I. (1992). The alteration of norepinephrine and acetylcholine concentration in immature rat urinary bladder caused by streptozotocin-induced diabetes. $J$. Urol. 148: 423-426.

Nilvebrant, L., Ekstrom, J. and Malmberg, L. (1986). Muscarinic receptor density in the rat urinary bladder after denervation, hypertrophy and urinary diversion. Acta Pharmacol. Toxicol. 59: 306314 .

Tammela, T.L.J., Leggett, R.E., Levin, R.M. and Longhurst, P.A. (1995).Temporal changes in micturition and bladder contractility after sucrose diuresis and streptozotocin-induced diabetes mellitus in rats. J. Urol. 155: 2014-2021.

Tong, Y.-C., Chin, Y.C. and Cheng, J.T. (2002). Alterations of $\mathrm{M}_{3}$-subtype muscarinic receptors in diabetic rat urinary bladder. Pharmacol. 64: 148-151.

Tong, Y.C., Hung, Y.C., Lin, S.N. and Cheng, J.T. (1996). Alterations in urinary bladder $\mathrm{M}_{2}$-muscarinic receptor protein and mRNA in 2-week streptozotocin-induced diabetic rats. Neurosci. Let. 206: $165-168$.

van Poppel, H., Stessens, R., Van Damme, B., Carton, L. and Baert, L. (1988). Diabetic cystopathy: neuropathological examination of urinary bladder biopsies. Eur. Urol. 15: 128-134. 